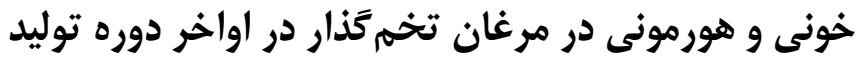

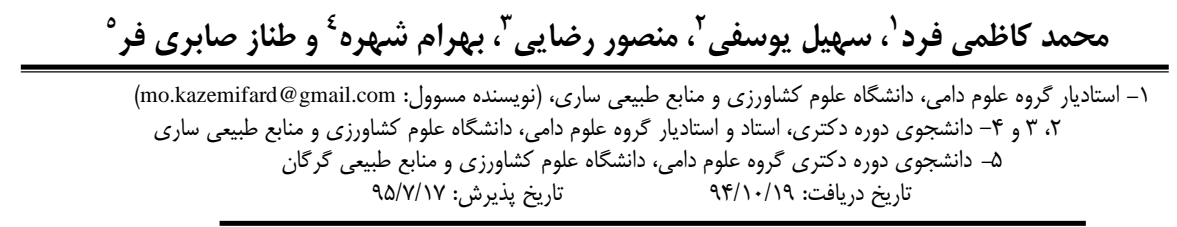

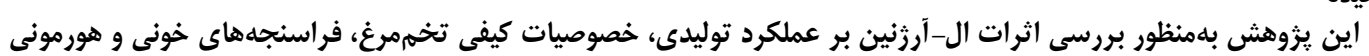

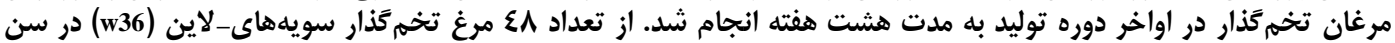

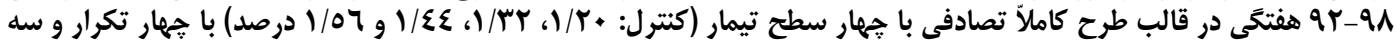

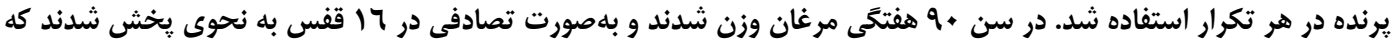

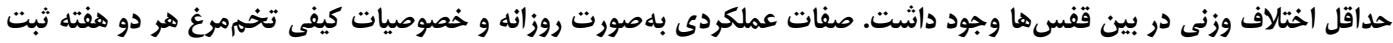

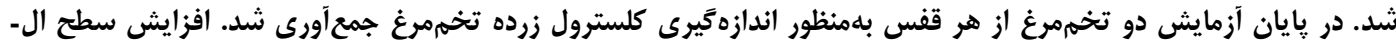

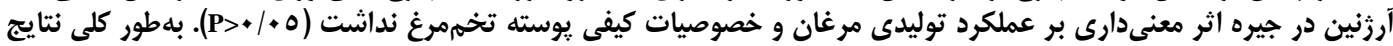

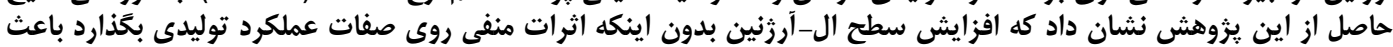

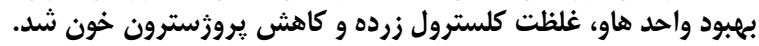

وازههاى كليدى: ضخامت يوسته تخممرغ، فراسنجه هور مونى، كلسترول زرده، مرغ تخم Fذار، واحد هاو، وزن مخصوص

كاهش مىيابد (آT) و از طرفى جون ال-آرزنين در بدن طيور

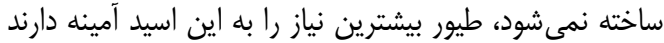

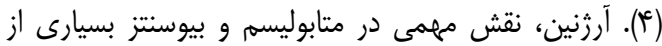

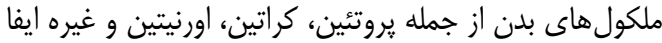

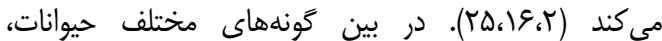

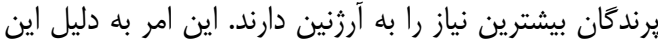

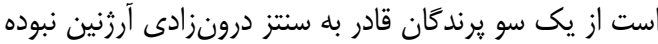

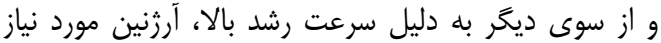

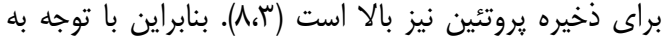

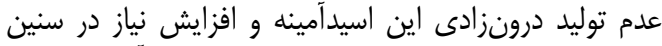

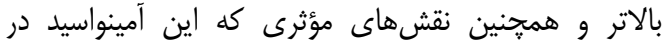

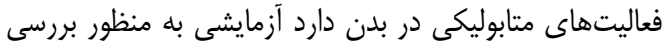

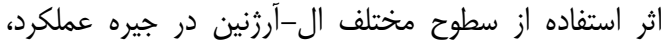

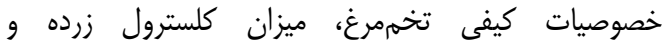

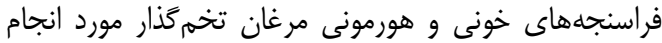

\section{مواد و روشها}

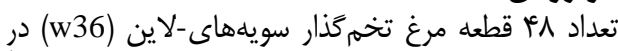

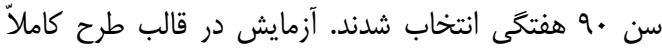

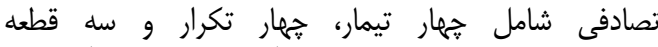

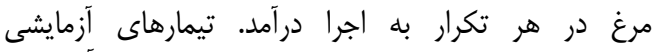

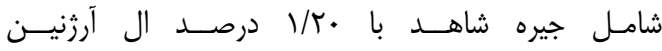
توصيه شده (Degussa Corporation, Kennesaw, GA)

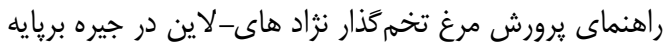

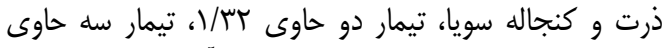

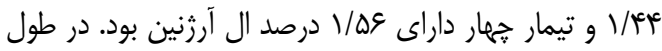

آرثنين بلهنوان يك اسيدآمينه ضرورى نقش بسيار

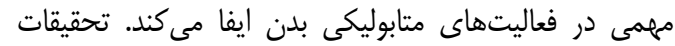

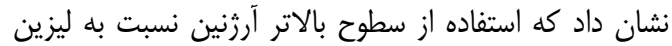

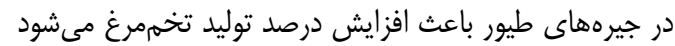

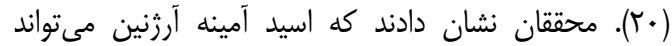

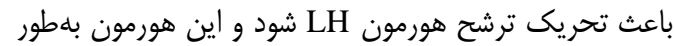

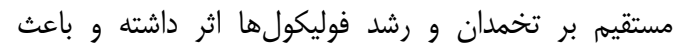

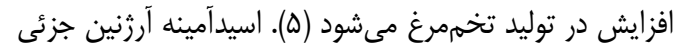

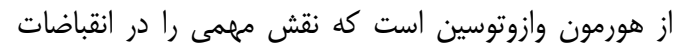

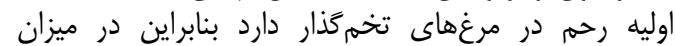

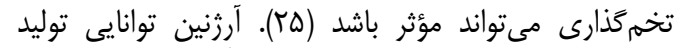

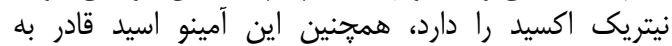

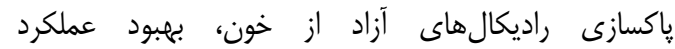

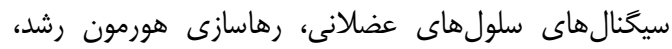

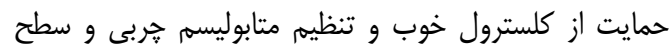

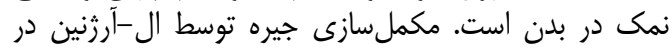

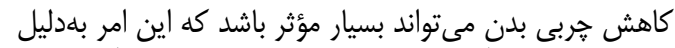

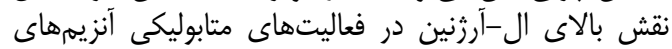

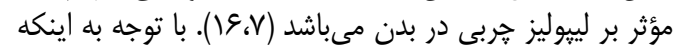

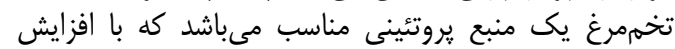

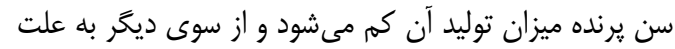

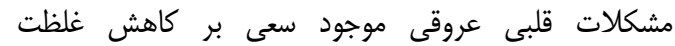

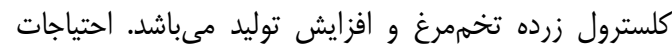

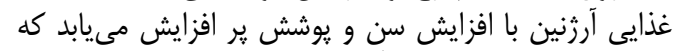

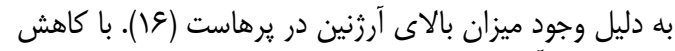

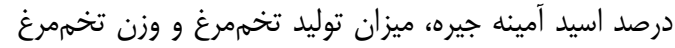




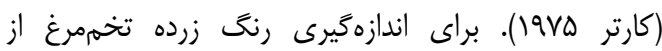

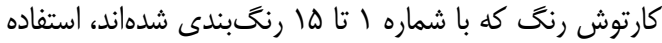

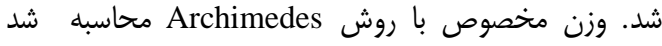

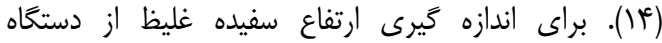

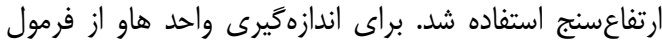

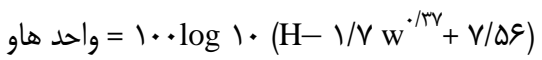

$$
\text { زير استفاده شد: }
$$

H : ارتفاع سفيده (ميلى

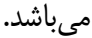

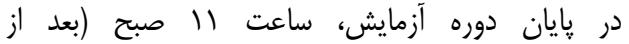

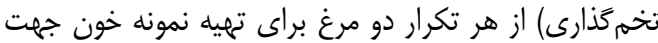

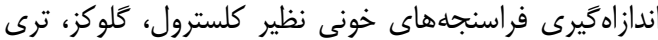

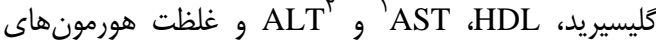

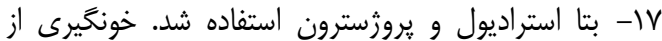

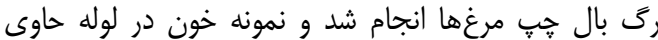
EDTA

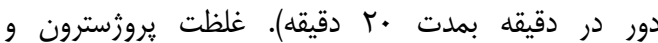

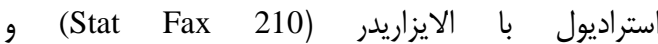

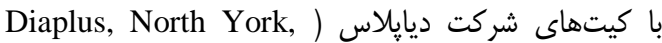
(Ontario, Canada كلوكز، ترى كليسيريد يلاسما با استفاده از دستخاه اتو آنالايزر

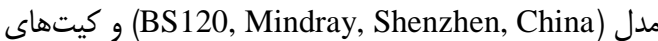

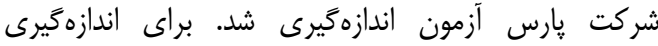

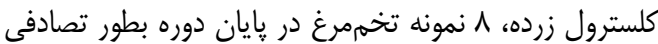

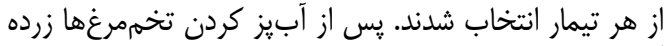

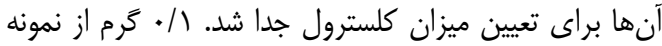

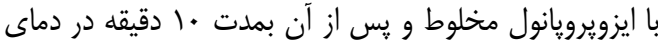

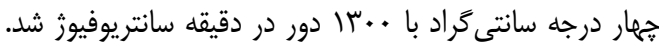

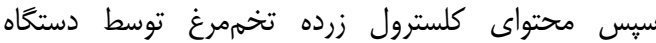

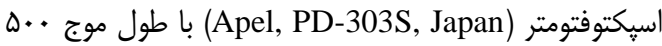

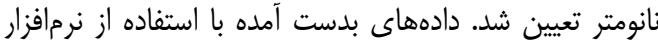

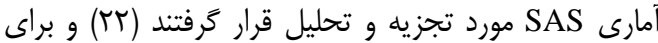

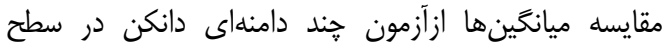

معنى دارى ينج درصد استفاده شد (1) (1).
دوره آزمايش آب و خوراك به طور آزاد در اختيار مرغهاى قرار

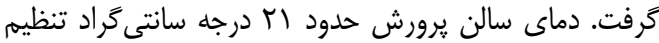

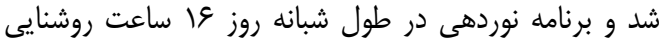

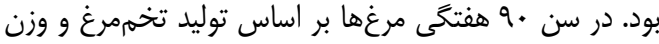

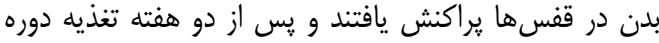

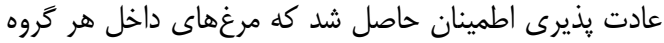

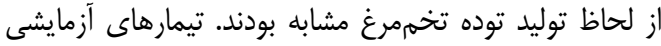

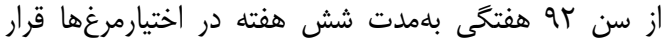
كرفتند. جيرهها براى گَروههاى مختلف آزمايشى بر اساس احتياجات

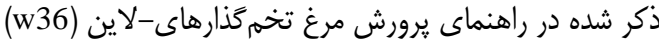

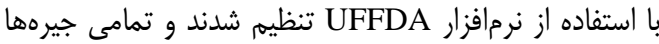
از لحاظ محتواى انرزى، يروتئين و ساير مواد مغذى يكسار نئن

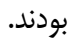
در طول آزمايش مصرف خوراك هر دو هفته اندازهخيرى و تخو تخون

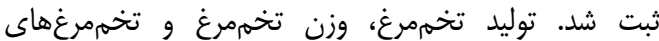

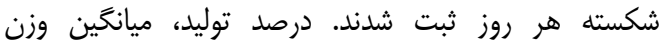

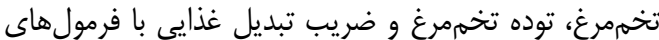

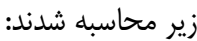

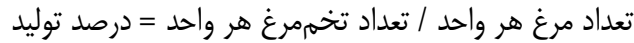

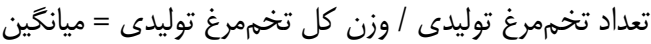

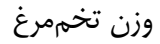

• 1 / درصد توليد × ميانگين وزن تخممرغ = توده تخمرمرغ

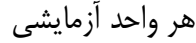

توده تخممرغ هر واحد / مصرف خوراك هر واحد = ضريب تبديل خوراى

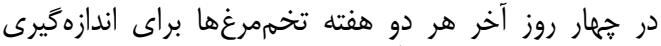

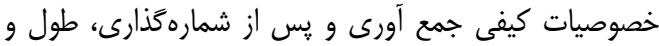

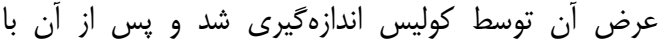

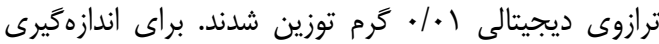

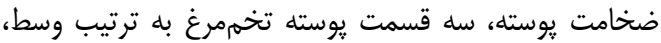

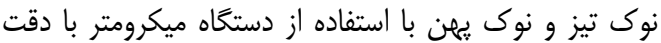

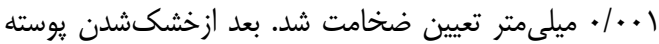

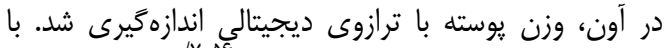

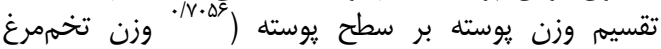

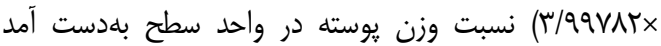




\begin{tabular}{|c|c|c|c|c|}
\hline \multicolumn{4}{|c|}{ تيمارها } & \multirow[t]{2}{*}{ 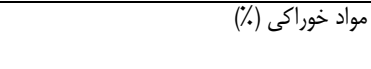 } \\
\hline r & r & 1 & شاهد & \\
\hline $91 / 99$ & $91 / 99$ & $91 / 99$ & $91 / 99$ & ذرت \\
\hline$r T / \cdot r$ & 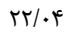 & $r T / \cdot r$ & $t r / . t$ & كنجاله سويا \\
\hline $1 . / \mathrm{AV}$ & $1 . / \mathrm{AV}$ & $1 . / \mathrm{AV}$ & $1 . / \mathrm{AV}$ & كربنات كلسيم \\
\hline $1 / 49$ & $1 / \% q$ & $1 / 49$ & $1 / r q$ & 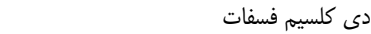 \\
\hline$\cdot / 41$ & $\cdot / 41$ & $\cdot / 41$ &.$/ 41$ & نمك \\
\hline r & r & r & r & 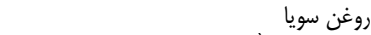 \\
\hline$\cdot / \pi \Delta$ & ./TQ & $\cdot / \pi \Delta$ & $\cdot / 4 \Delta$ & 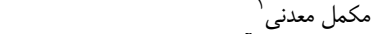 \\
\hline$\cdot / \pi \Delta$ & •/TS & $\cdot / \pi \Delta$ & $\cdot / 4 \Delta$ & 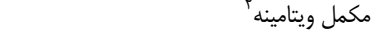 \\
\hline .11 &.$/ 1$ & .11 & . / & تركيب شيميايي محاسبه شده (٪) \\
\hline rNIF & rAlF & rNIF & rAlf & انرزى قابل متابوليسم (كيلو كالرى بر كيلوكرم) \\
\hline $10 / 19$ & $10 / \cdot 1$ & $10 / .+\%$ & $\mid Q / \cdot$. & 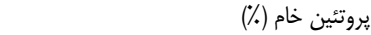 \\
\hline.$/ 4$ & $\cdot / 4$ &.$/ 4$ & $\cdot / 4$ & فسفر قابل دسترس (†) \\
\hline$r / \Delta$ & $F / \Delta$ & $r / \Delta$ & $r / \Delta$ & 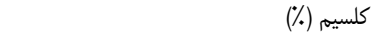 \\
\hline $1 / \Delta S$ & $1 / 44$ & $1 / \mu r$ & $1 / \pi \cdot$ & 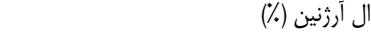 \\
\hline . & 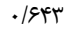 & 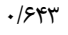 & 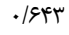 & متيونين + سيستئين (\%) \\
\hline - / AFq & - /AFq & - / A $F q$ & - /AFq & ليزين (\%) (ب) \\
\hline
\end{tabular}

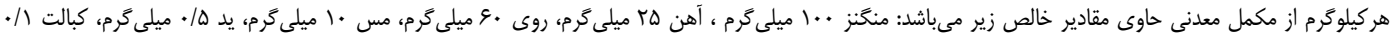

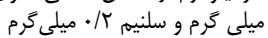

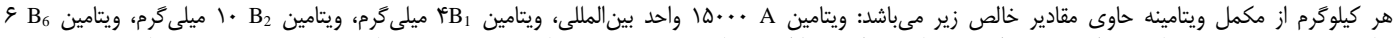

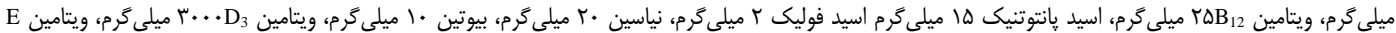
ا 10

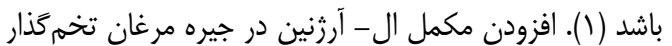

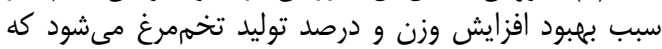

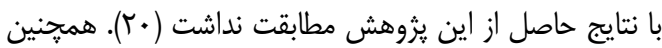

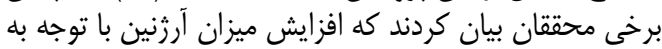

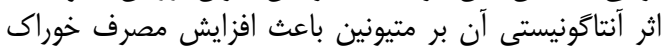

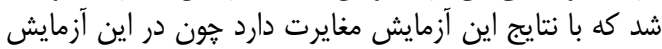

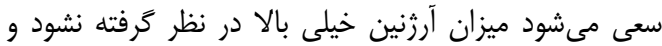

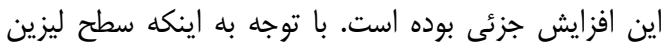

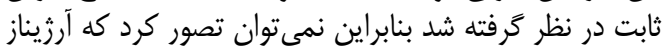

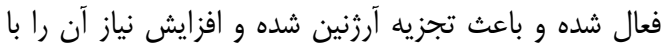
مصرف خوراك باعث شده است (و). افزايش سطح استر اسيد آمينه

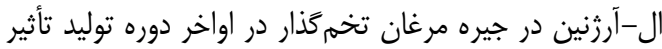

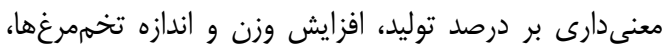

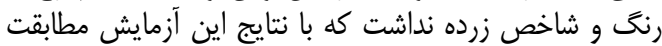

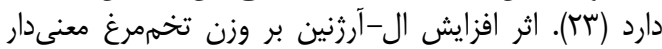

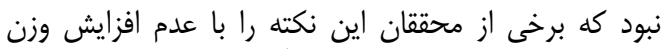

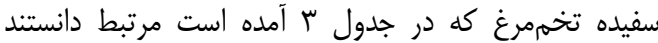

( $(\tau \cdot)$

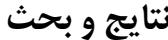

استفاده از سطوح مختلف اسيد آمينه آرخنين در جيره

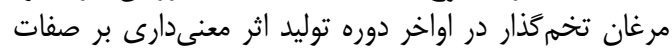

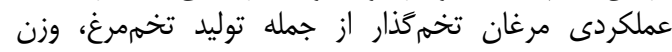

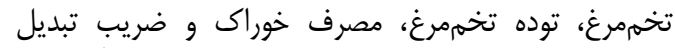

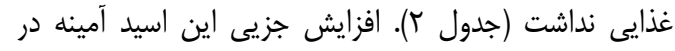

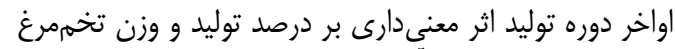

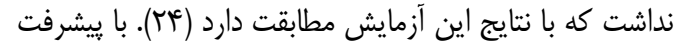

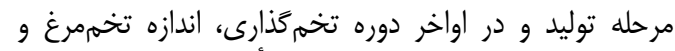

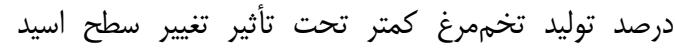

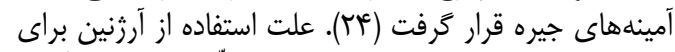

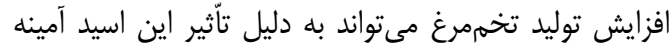

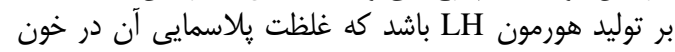

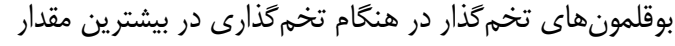

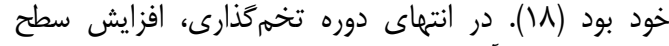

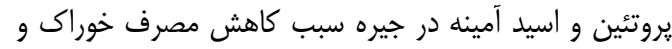

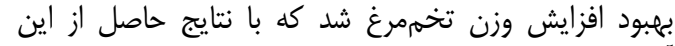

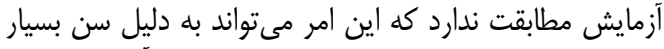

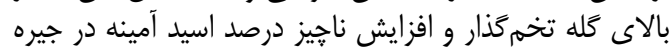




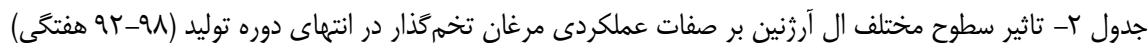
Table 2. Effect of different levels of L-Arginine on productive performance in laying hens at late phase of production (92-98 week)

\begin{tabular}{|c|c|c|c|c|c|c|}
\hline 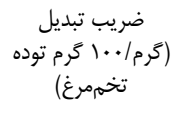 & (كرم/ يرنده در روز) & شاخص شكل تخممرغ & 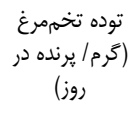 & 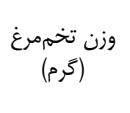 & توليد تخممرغ & \\
\hline$r / \cdot \varphi$ & $1 \cdot V / I 1$ & $V r / T f$ & $r \Delta / r)$ & $9 \% / V \cdot$ & $\Delta S / T \Delta$ & شاهد \\
\hline r/Tq & $1.9 / 4 \Lambda$ & $v e / r \Delta$ & D & $8 / / 4$. & GD/FV & تيمار r \\
\hline$r / 19$ & $111 / \cdot 1$ & $V E / r \Lambda$ & $r e / \varepsilon$. & $g r / v 1$ & V./F & تيمار ب \\
\hline T/Tr & $11 \% / 99$ & $V t / \Delta e^{c}$ & $M F / . r$ & $91 / 94$ & SV/AD & تيمار f \\
\hline$\cdot / \Delta \cdot$ & $\cdot / \Lambda 8$ &.$/ \mathrm{V} 9$ &.$/ \Delta F$ &.$/ \mathrm{q} 9$ &.$/ 14$ & سطح معنى دارى \\
\hline \multicolumn{7}{|c|}{ 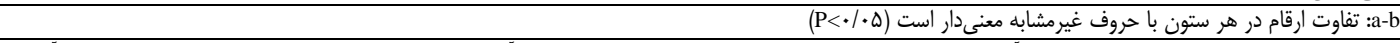 } \\
\hline
\end{tabular}

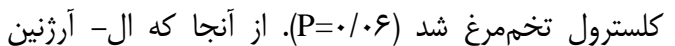

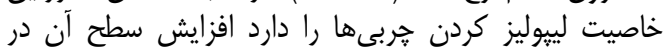

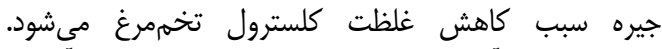

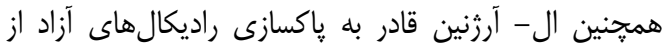

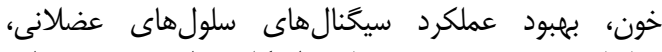

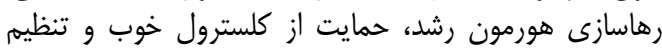

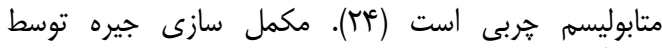

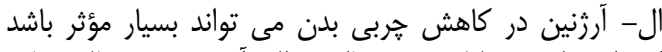

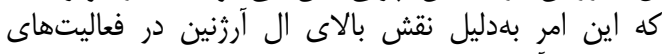

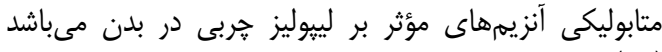

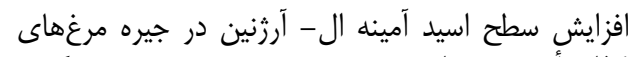

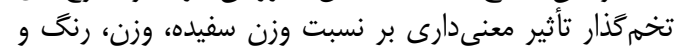

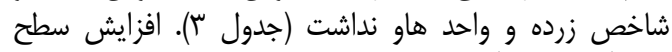

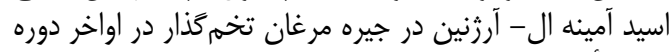

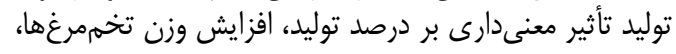

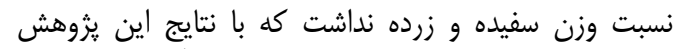

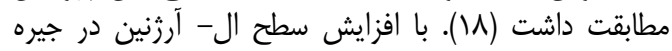

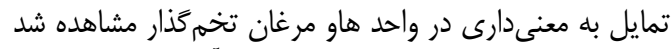

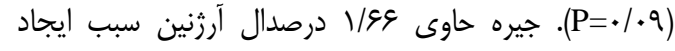

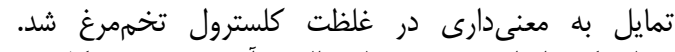

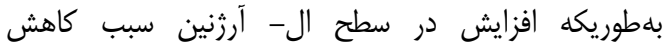

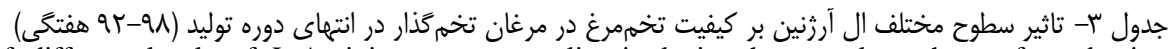
Table 3. Effect of different levels of L-Arginine on egg quality in laying hens at late phase of production

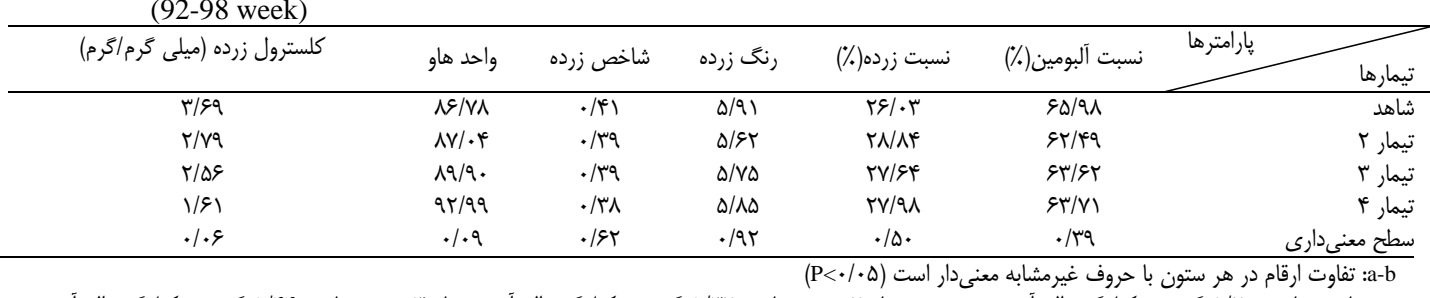

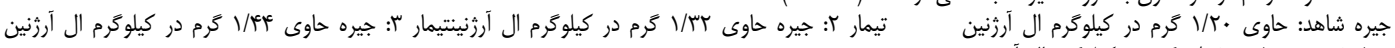

مىشود و اما در يثوهش حاضر افزايشى در اندازه و وزن إنان

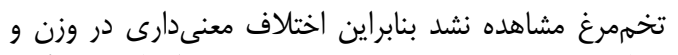

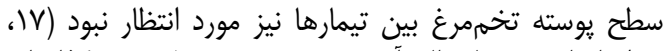

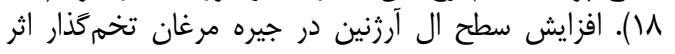

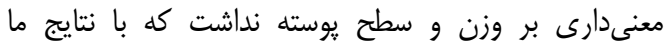

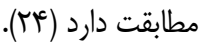

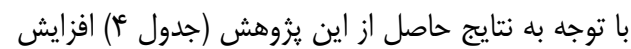

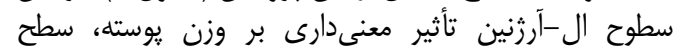

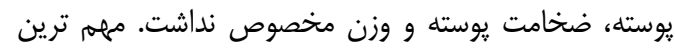

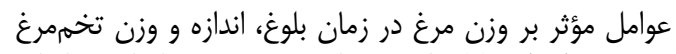

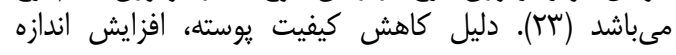

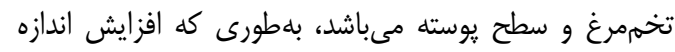

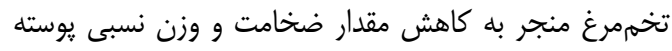

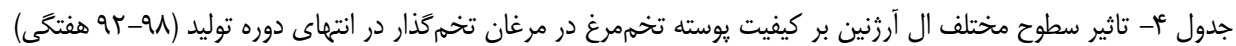
_Table 4. Effect of different levels of L-Arginine on egg shell quality in laying hens at late phase of production (92-98 week)

\begin{tabular}{|c|c|c|c|c|c|}
\hline وزن مخصوص & ضخامت يوسته & 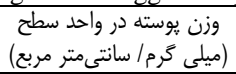 & 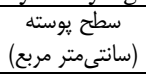 & $\begin{array}{l}\text { نسبت وزن يوسته } \\
\text { (\%) }\end{array}$ & تيمارها \\
\hline $1 / \cdot V$ & . & SN/TK & $V \Delta / \cdot f$ & V/aV & شاهد \\
\hline $1 / .9$ & 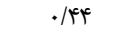 & $V \pi / 8 \Lambda$ & $v e / 1 \Delta$ & $N / \& \Delta$ & تيمار r \\
\hline $1 / \cdot v$ &.$/ 44$ & $V D / I V$ & $\mathrm{VQ} / \mathrm{AF}$ & $N / V T$ & تيمار r \\
\hline $1 / \cdot V$ & אז/. & $V . / 9 T$ & $V \in / A r$ & $N / r$. & تيمار \& \\
\hline $.1 \Delta \Delta$ &.$/ 94$ &.$/ D F$ & .199 &.$/ 19$ & سطح معنى دارى \\
\hline
\end{tabular}


نتيجه آن تخريب بافت مىباشد (·r). كه اين نتايج با نتايج

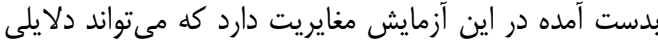

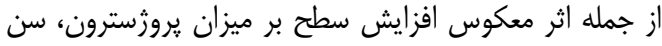

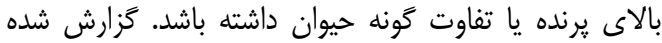

تزريق يروزسترون به مرغان تخمثذار تأثيرى بر كلوكز،

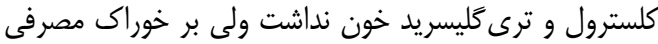

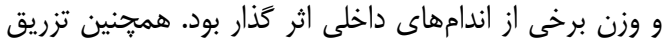

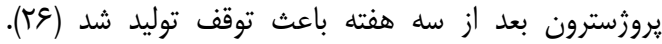

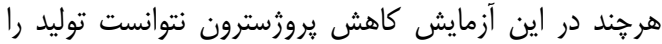

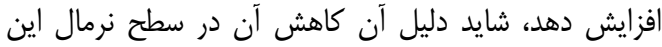

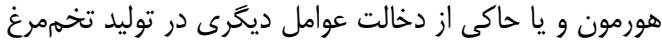

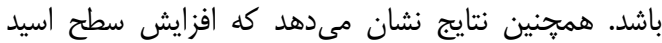

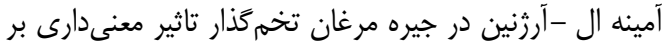

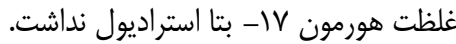

افزايش سطح ال-آرزنين در جيره اثر معنىدارى بر غلظت دارت

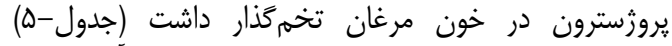

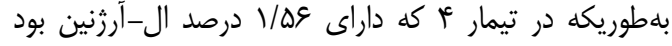

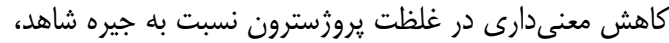

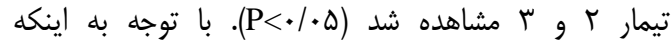

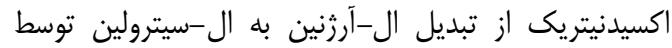

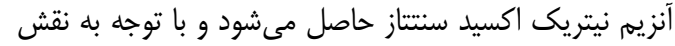

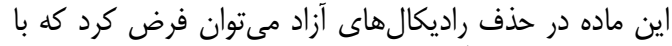

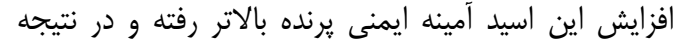

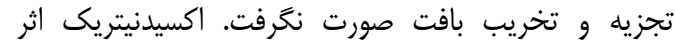

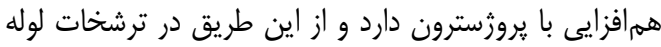

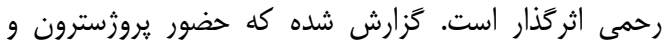

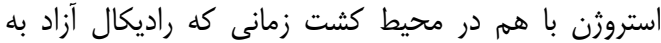

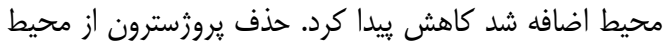

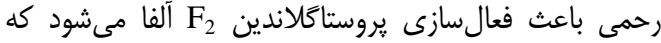

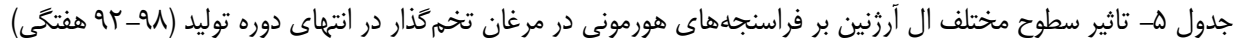
Table 5. Effect of different levels of L-Arginine on hormones in laying hens at late phase of production (92-98 week)

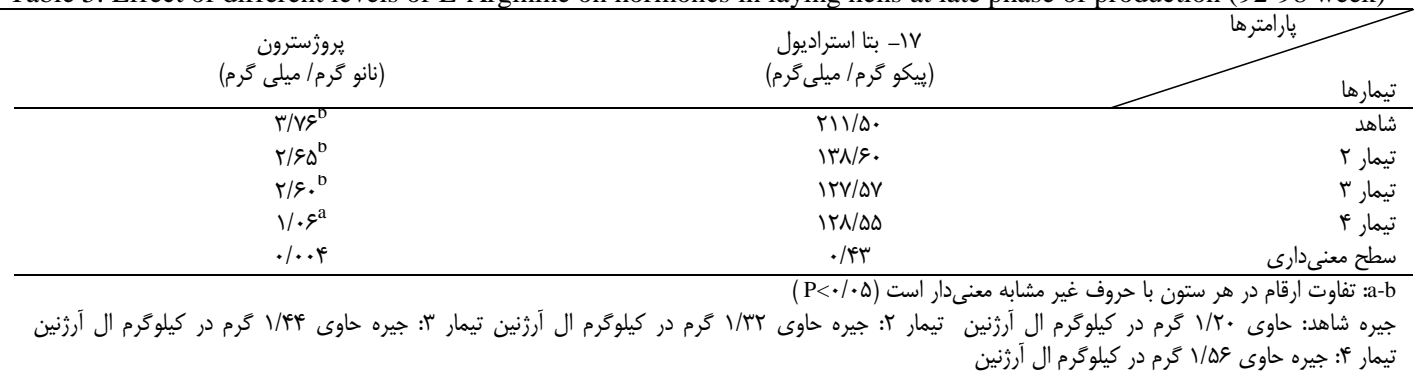

افزايش و نه درجهت كاهش مشاهده نشد (V). افزودن درصد

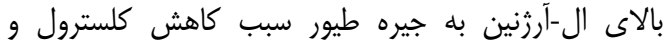

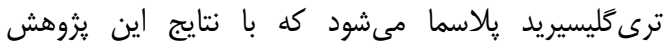

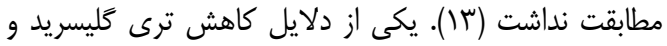

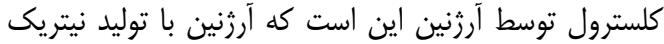

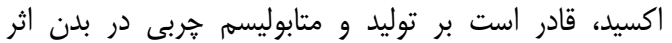

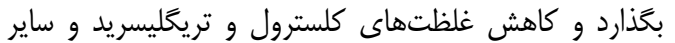

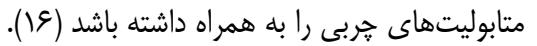

با توجه به نتايج حاصل از اين :ثروهش (جدول ع) افزايش

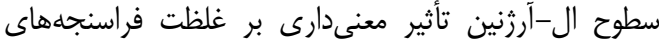

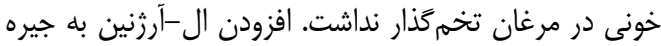

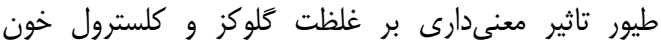

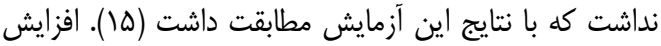

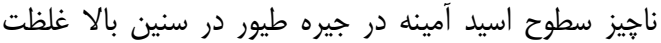

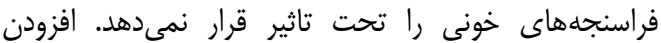

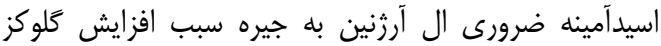
خون مىشود اما در اين تحقيق اثر معنىدارى إنه نه در جهت

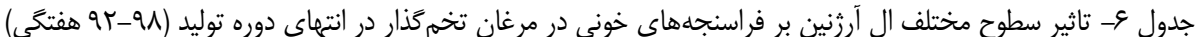
Table 6. Effect of different levels of 1-Arginine on blood parameters in laying hens at late phase of production (92-98 week)

\begin{tabular}{|c|c|c|c|c|c|c|}
\hline $\begin{array}{l}\text { ALT } \\
\text { (واحد بين) } \\
\text { (الملى) }\end{array}$ & $\begin{array}{c}\text { AST } \\
\text { (والحد بين) } \\
\text { (الملى) }\end{array}$ & 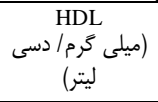 & (ميلى كرم/ دسىى ليتر) & (ميلى گرم/ دستى ليتر) & (ميلى كرم/ دلى دسى & \\
\hline$r / V \Delta$ & $I V Q / \Delta$ & sT/VD & $179 / / 4$ & $1 \cdot \Delta / r \Delta$ & TIT/VQ & شاهد \\
\hline$\Gamma / \Delta$ & ISN/TQ & 91 & IVIT & $1 \mathrm{rq} / \mathrm{gV}$ & rIF/VQ & تيمار r \\
\hline F/VQ & $19 \cdot / \pi \Delta$ & $s \mathrm{~V} / \mathrm{V} \Delta$ & $|F V| / V$ & let & $r \cdot g / \Delta$. & تيمار ץ \\
\hline f & $\mid \wedge q / \Gamma \Delta$ & v. & & $|r| / g V$ & $r \cdot \Delta / \Delta$. & تيمار F \\
\hline. $\mid a 4$ & .14. & $\cdot / V T$ & .190 & Tr/. & . & سطح معنى دارى \\
\hline
\end{tabular}

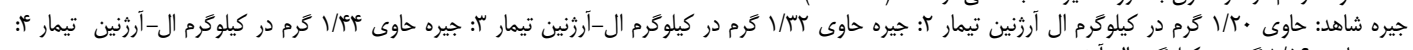
جيره حاوى \&ه/ا كَرم در كيلوكَرم ال آررثنين

فراسنجههاى خونى مرغان تخمثزار در اواخر دوره توليد

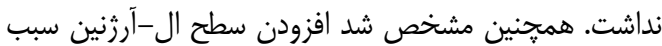

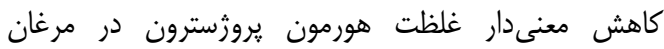

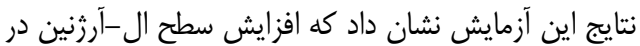

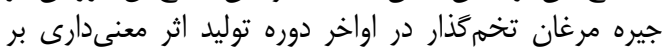

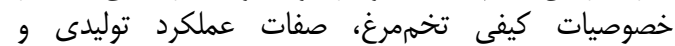




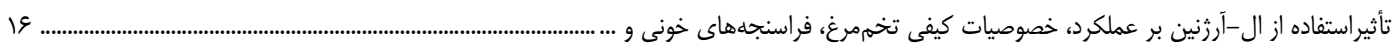

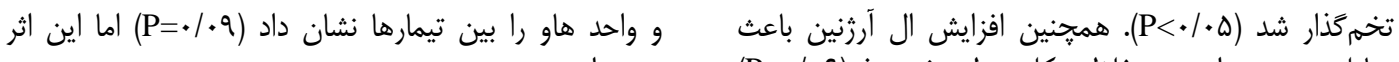

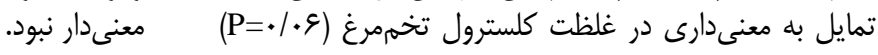
antioxidants capacity, Lipid peroxidation, nitric oxide, egg weight and blood biochemical values in japanase quails. Biological Trace Element Research,132: 136-143.

2. Austic, R.E. and M.C. Nesheim. 1972. Arginine and creatine interrelationships in the chick. Poultry Science, 51: 1098-1105.

3. Baker, D. H. 2009. Advances in protein-amino acid nutrition of poultry. Amino Acids, 37: 29-41.

4. Ball, R.O., K.L. Urschel and P.B. Pencharz. 2007. Nutritional consequences of interspecies differences in arginine and lysine metabolism. Journal of Nutrition, 137: 1626-1641.

5. Basiouni, G., H. Najib, M.M. Zaki and A.S. Al-Ankari. 2006. Influence of extra supplementation with arginine and lysine on overall performance, ovarian activities and humoral immune response in local saudi hens. International Journal of Poultry Science, 5: 441-448.

6. Basiouni, G.F. 2009. The effect of feeding extra amounts of arginine to Local Saudi Hens on luteinizing hormone secretion. Journal of Biological Sciences, 9: 617-620.

7. Boorman, K.N., I.R. Falconer and D. Lewis. 1968. The effect of lysine infusion on the renal reabsorption of arginine in the cockerel. Proceedings of the Nutrition Society, 27: 61-62A.

8. Coroz, A., J.R. Moran and D. Hoehler. 2003. Arginine need of heavy broiler males: applying the ideal protein. Poultry Science, 82402-407.

9. Corzo, A., M.T. Kidd, D.J. Burnham and B.J. Kerr. 2003. Dietary glycine needs of broiler chicks. Poultry Science, 83: 1382-1384.

10. Cui, H.X., M.Q. Zhang, R.R. Liu, G.P. Zhao, J.L. Chen and J. Wen. 2012. Liver dominant expression of fatty acid syntheses gene in two chicken breeders during intramuscular-fat developments. Molecular Biology Reports, 39: 3479-3484.

11. Duncan, D.B. 1955. Multiple Ranges and Multiple F-tests. Biometrics, 11: 1-42.

12. Ebrahimi, M., A. Zare-Shahne, Z. Ansari-Pirsaraie, M. Tabiyaniyan, M. Adibmoradi and K. Nuri-Jaliyani. 2013. Effect of dietary L-Arginine on growth performance and caracas characteristics during pre-starter period in broiler chickens. National Conference of Animal and Poultry. (In Persian).

13. Emadi, M., K. Kaveh, M.H. Bejo, A. Ideris, F. Jahanshiri, M. Ivan and R.A. Alimon. 2010. Growth performance and blood parameters as influenced by different levels of dietary arginine in broiler chickens. Journal of Animal and Veterinary Advance, 9: 70-74.

14. Hempe, J.M., R.C. Laukxen and J.E. Savage. 1988. Rapid determination of egg weight and specific gravity using a computerized data collection system. Poultry Science, 67: 902-907.

15. Jobgen, W., C.J. Meininger, S.D. Jobgen, P. Li, M.J. Lee, S.B. Smith, T.E. Spencer, S.K. Fried and G. Wu. 2009. Dietary L-arginine supplementation reduces white fat gain and enhances skeletal muscle and brown fat masses in diet-induced obese rats. Journal of Nutrition, 139: 230-237.

16. Khajal, F. and R.F. Widerman. 2010. Dietary arginine: metabolic, environmental, immunological and physiological interrelationships. World's Poultry Science Journal, 66: 751-766.

17. Khajali, F., M. Tahmasebi, H. Hassanpour, M.R. Akbari, D. Qujeq and R.F. Wideman. 2011. Effects of supplementation of canola mealbased diets with arginine on performance, plasma nitric oxide and carcass characteristics of broiler chickens grown at high altitude. Poultry Science, 10: 87-94.

18. Leeson, S. and J.D. Summers. 2001. Nutrition of the chicken. $4^{\text {th }}$ ed. Guelph: University Books.

19. Liu, H.K., D.W. Long and W.L. Bacon. 2002. Interval between preovulatory surges of luteinzing hormone increases late in the reproductive period in turkey hens. Biology of Reproduction, 66: 1068-1075.

20. Najib, H. and B. Ghazi. 2004. Determination of the nutritional requirements of the Local Saudi chickens: 1. Effect of Arginine inclusion, in excess of the leghorn requirement, on performance of the local Saudi chickens. Scientific Journal of King Faisal University 'Basic and Applied Sciences, 5: 131-144.

21. Nasiri-Moghadam, H., M. Kazemi Fard, M.J. Agah, S.J. Husseini and M.T. Mirakzehi. 2012. Effect of different levels of methionine, protein and tallow on the productive performance and egg quality of laying hens in the late-phase production. Brazilian Journal of Poultry Science, 12149-158.

22. SAS Institute. 2000. SAS 8/01. SAS Institute INC., Cary, NC.

23. Scott, M.L., M.C. Nesheim and R.J. Young. 1982. Nutrition of chicken. ML Scott and Associates publishers, Ithaca, NY.

24. Silva, L.M., A.E. Murakami, J.I. Fernandes, D. DallaRosa and J.F. Urgnani. 2012. Effects of dietary arginine supplementation on broiler breeder egg production and hatchability. Brazilian Journal of Poultry Science, 14: 267-273.

25. Takahashi, T., M. Kawashima, M. Kamiyoshi and K. Tanaka. 1994. Arginine vasotocin receptor bindingin the hen uterus (shell gland) before and afteroviposition. European Journal of Endocrinology, 130: 366-372.

26. Zaghari, M., R. Taherkhani and S.H. Honarbakhsh. 2009. Effects of progesterone injection on performance, plasma hormones and ovarian morphology of ad libitum and restricted fed broiler breeder hens. African Journal of Biotechnology, 8: 6481-6489. 


\title{
Effect of L-Arginine on Productive Performance, Egg Quality Parameters, Blood and Hormone Parameters of Laying Hens in Late-phase of Production
}

\section{Mohammad Kazemi-Fard ${ }^{1}$, Soheil Yousefi ${ }^{2}$, Mansour Rezaei ${ }^{3}$, Bahram Shohre ${ }^{4}$ and Tannaz Saberifar}

1- Assistant Professor, Department of Animal Science, Sari Agricultural Sciences and Natural Resources University, Sari, Iran (Corresponding author: Mo.kazemifard@gmail.com)

2, 3 and 4- PhD Student, Professor and Assistant Professor, Department of Animal Science, Sari Agricultural Sciences and Natural Resources University, Sari, Iran

5- PhD Student, Department of Animal Science, Gorgan Agricultural Sciences and Natural Resources University, Gorgan, Iran

Received: January 9, $2016 \quad$ Accepted: October 8, 2016

\begin{abstract}
This experiment was conducted to investigate effects of 1-arginine on productive performance and egg quality parameters, blood and hormone parameters of laying hens in late phase of production for 8 weeks. Forty eight Hy-Line W-36 were used in a completely randomize design with four level of 1-arginine $(1 / 20=$ control diet, 1/32, 1/44 and 1/56 percent) and four replicate from 92-98 weeks of age. Layers were weighed at 90 week of age and randomly distributed into 16 cages in order to have a similar pen body weight. The performance data were recorded daily, whereas the egg quality traits were determined biweekly. At the end of experiment 2 eggs from each cage were collected for egg yolk cholesterol analysis. Supplementation of l-arginine in diet did not have significant effect on productive performance and egg shell quality in layers. Furthermore, the addition of 1-arginine to diet did not have significant effect on the egg yolk cholesterol (P>0.05). The results of this study showed that supplementation of 1arginine improved Hough unit and yolk cholesterol and blood progesterone with no adverse effect on productive performance.
\end{abstract}

Keywords: Egg Shell Thickness, Hormone Parameters, Hough Unit, Layer Hen, Specific gravity, Yolk Cholesterol 